\title{
Study of damages induced on ATLAS silicon by fast extracted and intense proton beam irradiation
}

\section{Bertella ${ }^{a * \dagger}$}

${ }^{a}$ Institute of High Energy Physics, Chinese Academy of Sciences, Beijing, China

E-mail: claudia.bertella@cern.ch

C. Escobar ${ }^{b}$, J. Fernández-Tejero ${ }^{c}$, C. Fleta $^{c}$, G. Gariano $^{d}$, A. Gaudiello ${ }^{d}$, C. Gemme $^{d}$, S. Katunin ${ }^{e}$, A. Lapertosa ${ }^{d}$, M. Miñano $^{b}$, A. Rovani ${ }^{e}$, E. Ruscino ${ }^{e}$, A. Sbrizzi ${ }^{f}, \mathbf{M}$. Ullán ${ }^{c}$

a Institute of High Energy Physics (IHEP), Chinese Academy of Sciences (CAS), Beijing, China,

${ }^{b}$ Instituto de Fisica Corpuscular (IFIC), Centro Mixto Universidad de Valencia (CSIC), Spain,

${ }^{c}$ Centro Nacional de Microelectrónica, (IMB-CNM), CSIC, Barcelona, Spain,

${ }^{d}$ Istituto Nazionale di Fisica Nucleare (INFN), Sez. Genova, Genova, Italy,

${ }^{e}$ Petersburg Nuclear Physics Institute (PNPI), St. Petersburg, Russia,

${ }^{f}$ Istituto Nazionale di Fisica Nucleare (INFN), Sez. Bologna, Bologna, Italy

\begin{abstract}
The ATLAS silicon tracker detectors are designed to sustain high dose integrated over several years of operation. This very substantial radiation hardness should also favour the survival of the detector in case of accidental beam losses. An experiment performed in 2006 showed that ATLAS pixel detector modules (silicon planar hybridly coupled with FE-I3 electronics) could survive to beam losses up to $1.5 \cdot 10^{10}$ protons $/ \mathrm{cm}^{2}$ in a single bunch with minimal or no deterioration of performance. The upgrade of LHC to even higher luminosity (HL-LHC) calls for a new test of these properties. Two test beam campaigns have been done in 2017 and 2018 at the HighRadiation to Materials (HiRadMat) Facility of the CERN Super Proton Synchrotron in order to establish for the first time the damage threshold of different types of ATLAS IBL pixel and ITk strip detectors under very intense proton beam irradiation.
\end{abstract}

The 27th International Workshop on Vertex Detectors - VERTEX2018

22-26 October 2018

MGM Beach Resorts, Muttukadu, Chennai, India

\footnotetext{
* Speaker.

$\dagger$ The authors would like to thank the HiRadMat operation team for the technical support during the experiment. This project is co-funded by ARIES in the frame of the European Union's Horizon 2020 Research and Innovation programme under Grant Agreement no. 730871.
} 


\section{Introduction}

The Large Hadron Collider is the word largest hadron accelerator, which is providing since 2010 high energy proton-proton collisions in four interaction points along its ring. The first one is surrounded by the ATLAS detector [1]. This multipurpose detector was built to face high luminosity operations, with a design instantaneous luminosity of $10^{34} \mathrm{~cm}^{-2} \mathrm{~s}^{-1}$. Its Inner Detector (ID) was tailored to sustain a high dose integrated over several years of operation. Even though the level of radiation hardness of the ID is in favour of the detector survival in case of beam failures, a dedicated study has been recently performed to better understand the impact of a beam loss in the vicinity of the ATLAS detector. A similar study was already performed in 2006 [2]. This experiment was able to establish that the pixel modules could survive to beam losses as large as $1.5 \cdot 10^{10}$ protons $/ \mathrm{cm}^{2}$ in a single bunch of $42 \mathrm{~ns}$, with minimal or no deterioration of performance. In 2025 the High Luminosity LHC (HL-LHC) [3] will replace the LHC. The upgraded accelerator allows to increase the nominal LHC instantaneous luminosities by a factor of five $\left(7.5 \cdot 10^{34} \mathrm{~cm}^{-2} \mathrm{~s}^{-1}\right)$, and thereby enabling the experiments to enlarge their data sample by one order of magnitude $\left(4000 \mathrm{fb}^{-1}\right)$. In order to increase the luminosity, the apertures in the focusing sections around the experiments will be enlarged with respect to the LHC configuration. Moreover, the aperture of the quadrupole absorbers (XTAS) protecting the magnets from the collision debris will be also extended potentially exposing the ATLAS detectors to a larger fluence of beam-induced radiation towards the interaction region. To cope with harsh condition expected for HL-LHC, a major upgrade of the all the ATLAS sub-systems is planned. The ID will be replaced with a full silicon Inner Tracker (ITk) with increased granularity and radiation hardness [4,5]. In view of the HL-LHC, it is vital to perform new measurement of the beam losses survival threshold. The ID survival to LHC beam losses can be tested by exposing single module to beam intensities which are comparable or larger then those expected in case of a "pilot beam" loss. This is possible using the CERN Super Proton Synchrotron (SPS) fast extracted proton beam, as it will be discussed in the next section. Preliminary results on this topic can be found in the following Ref. [6].

\section{The HiRadMat Facility at CERN}

High-Radiation to Materials (HiRadMat) [7] is a test beam facility at CERN, which provides high-intensity pulsed beams to an irradiation area where material samples can be tested. The facility extracts high-energy proton beam $\left(440 \mathrm{GeV} / c^{2}\right)$ from the CERN SPS. Each pulse can include from 1 to 288 bunches with $25 \mathrm{~ns}$ separation, reaching a pulse length of $7.2 \mu$ s. Two separate parallel tunnels, characterized by different radiation level, host the beam line with the experimental tables (TNC tunnel) and the read-out system (TT61 tunnel). Long connecting cables ( $\sim 15 \mathrm{~m}$ ) are passed through a concrete screening wall placed between the two tunnels to connect the experiment to the read-out system and power supply. Due to the high radiation levels, the TNC and TT61 tunnel cannot be access during the operations. Fully remote controlled system is required by the facility.

\section{Experimental setup}

Two beam test campaigns have been performed in July 2017 and May 2018. In 2017, the experiment was installed downstream of the collimator prototype for HL-LHC (called RotColl). 
However, the first day of operations was fully dedicated to the module irradiation. A week of RotColl operations followed the first shift. Finally, one last day was devoted to this experiment. In May 2018, the module irradiation was the main experiment and the shift was fully devoted to its operations.

\subsection{Test box}

The test box, made with epoxy, fibreglass, makrolon, and aluminium, was designed to host up to 8 modules on dedicated frames. It was provided with a cooling system composed of four fans to allow air flow and keep the temperature on the modules close to $40{ }^{\circ} \mathrm{C}$. The three tested modules were loaded on fibreglass frames within the test box and aligned with the beam position. The proton beam was hitting the modules perpendicularly, unlike to the previous test [2]. In May 2018, a few improvements were deployed on the test box and module frames:

- the modules have been isolated by the light thanks to an opaque shielding;

- the fans were remotely controlled to improve the air flow;

- the box was placed on a moving table to put the module in and out of the beam;

- a heat dissipator and an aluminium heat sink were added on the pixel frame to reduce the temperature;

- aluminium foils were placed in the front and back of the box to provide beam position.

\subsection{Modules}

The newest generation of ATLAS pixel modules has been tested: 3D [8] and planar [9] Insertable B-Layer (IBL [10]) sensor. The sensor is connected to front-end (FE)-I4 [11] chip which was designed in $130 \mathrm{~nm}$ CMOS technology in order to cope with higher radiation levels (250 MRad) and larger occupancies. In 2017, 2 IBL 3D modules were tested using different configurations to reproduce the standard status of the detectors when LHC is delivering stable beams (sensor bias and FE amplifiers on) and non-stable beams (sensor bias and FE preamplifier off). Two different ITk strip miniature modules were tested: ATLAS12 [12] and PTP (Punch-ThroughProtection) $[13]^{1}$. The $\mathrm{PTP}^{2}$ structure is meant to protect the strip integrity for high charge deposition. Both sensors were wire bonded to an ABC130 read-out chip [14]. Table 1 summarises the major modules characteristics for both beam test campaigns.

\subsection{Beam operation}

Two beam radius were used in both beam tests: $2 \mathrm{~mm}$ and $0.5 \mathrm{~mm}$. The latter was meant for testing the local irradiation while the former for global irradiation. Table 2 summarises the number of bunches request in each configuration, as well as the beam intensity and the total number of protons seen by the modules.

\footnotetext{
${ }^{1}$ In 2018, a second ITk strip miniature module without PTP structure was irradiated. However, due to some technical issues, it was not possible to monitor the performance during the beam test. Therefore, no results on this module have been included in this report.

${ }^{2}$ The distance between the implant and the bias ring is about $20 \mu \mathrm{m}$.
} 


\begin{tabular}{|c|c|c|c|c|c|c|}
\hline Module & $\begin{array}{l}\text { Total } \\
\text { size }\end{array}$ & $\begin{array}{l}\text { Sensor } \\
\text { type }\end{array}$ & $\begin{array}{c}\text { Sensor } \\
\text { thickness }\end{array}$ & $\begin{array}{l}\text { Chip } \\
\text { type }\end{array}$ & Channel (pitch) & $\begin{array}{c}\text { Maximum } \\
\text { dose }\end{array}$ \\
\hline \multicolumn{7}{|c|}{ Beam test 2017} \\
\hline IBL & $2 \times 2 \mathrm{~cm}^{2}$ & $\mathrm{n}^{+}$-in-p, 3D [8] & $230 \mu \mathrm{m}$ & FE-I4 [11] & $26680\left(50 \times 250 \mu \mathrm{m}^{2}\right)$ & $250 \mathrm{MRad}$ \\
\hline ITk & $1 \times 1 \mathrm{~cm}^{2}$ & $\mathrm{n}^{+}$-in-p, ATLAS12 [12] & $320 \mu \mathrm{m}$ & $\mathrm{ABC} 130[14]$ & $104(74.5 \mu \mathrm{m})$ & $35 \mathrm{MRad}$ \\
\hline \multicolumn{7}{|c|}{ Beam test 2018} \\
\hline IBL & $2 \times 4 \mathrm{~cm}^{2}$ & $\mathrm{n}^{+}$-in-n, planar [9] & $200 \mu \mathrm{m}$ & FE-I4 [11] & $2 \times 26680\left(50 \times 250 \mu \mathrm{m}^{2}\right)$ & $250 \mathrm{MRad}$ \\
\hline ITk & $0.7 \times 2.6 \mathrm{~cm}^{2}$ & $\mathrm{n}^{+}$-in-p, PTP [13] & $300 \mu \mathrm{m}$ & $\mathrm{ABC} 130$ [14] & $64(77 \mu \mathrm{m})$ & $35 \mathrm{MRad}$ \\
\hline
\end{tabular}

Table 1: Summary of the modules' characteristics used in both beam tests.

\begin{tabular}{|c|c|c|c|c|c|}
\hline Name & Beam radius & Spacing & Bunches & Proton intensity & Total protons \\
\hline \multicolumn{6}{|c|}{ Beam test 2017} \\
\hline global irradiation & $2 \mathrm{~mm}$ & $25 \mathrm{~ns}$ & $1,4,12,24,36,72,144,288$ & $10^{10}\left(10^{11}\right)$ & $3.8 \cdot 10^{13}$ \\
\hline local irradiation & $0.5 \mathrm{~mm}$ & $25 \mathrm{~ns}$ & $1,12,72,288$ & $10^{11}$ & $3.7 \cdot 10^{13}$ \\
\hline \multicolumn{6}{|c|}{ Beam test 2018} \\
\hline global irradiation & $2 \mathrm{~mm}$ & $25 \mathrm{~ns}$ & $1,4,12,24,36,72,144,288$ & $10^{11}$ & $1.16 \cdot 10^{13}$ \\
\hline local irradiation & $0.5 \mathrm{~mm}$ & $25 \mathrm{~ns}$ & 1,12 & $10^{11}$ & $2.6 \cdot 10^{12}$ \\
\hline
\end{tabular}

Table 2: Summary of the proton beam pulse list in both beam tests. Beam radius, number of bunches, proton intensity and total protons received by the modules are presented.

\subsection{Test routine}

The modules were tested during beam inter-fill. On all IBL modules, the sequence below was followed:

- current versus voltage bias (IV) scan to monitor the leakage current at different bias voltage;

- digital and analog scan: check the FE analog and digital functionality;

- threshold scan: measure the occupancy at different injected charges for a fixed threshold $\left(3000 e^{-}\right)$. The response curve may be fitted with an error function (ERF), where the slope is a characteristic of the noise;

- self-triggering scan: the outputs of the individual pixel comparators are ORed together to form a Hitbus signal that is used as a trigger to acquire and record events.

While on the ITk, the following checks were performed:

- leakage current continuously monitored online with the detector under bias;

- three points gain: injecting three different charges and varying the threshold value of the discriminator from zero to its maximum. The measured average hit rate versus threshold is fitted with a sigmoidal curve and the value at its 50\% (Vt50) is extracted as well as it sigma. From a linear fit of charge versus $V t 50$, the noise and gain can be obtained.

During the operations, the IBL modules' configuration was lost after each pulse, but the normal detector operation was recovered with a reconfiguration, up to 72 bunches with $0.5 \mathrm{~mm}$ radius in 2017 beam test. However, the last shot in 2017 with 288 bunches with $0.5 \mathrm{~mm}$ radius caused a short circuite in FE-I4 at the pixel level in both IBL 3D modules, thereby it was not possible to operate them anymore. 


\section{Results}

\subsection{IBL pixel modules}

Preliminary results of the two beam tests on the IBL pixel modules are presented in this section. Figure 1 (a) and (b) shows the noise map for the 3D module in stable-beam and standby configuration, respectively. The noise scan for the planar module is presented in Figure 1 (c). For a non irradiated module, the noise value is about $130 e^{-}$(ENC). In both 3D and planar modules, it is visible that after the irradiation the noise increases in correspondence of the beam spot up to $300 e^{-}$. With the self-triggering scan, it was also noticed that the part of the detector hitted by the beam was activated. A linear correlation was also observed between the degradation of the performace of the modules and the proton fluence ${ }^{3}$ received by the same. The perfomance was measured in term of noise level per pixel. The level of the leakage current ${ }^{4}$ of the sensors was also moni-

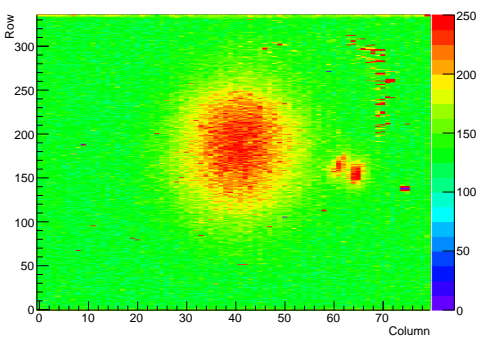

(a)

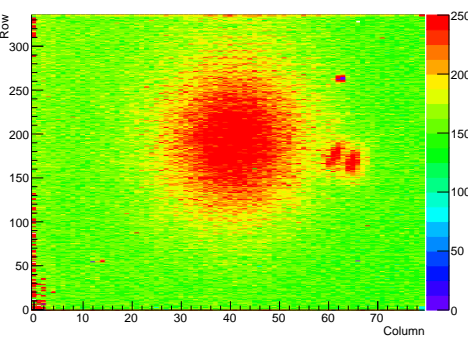

(b)

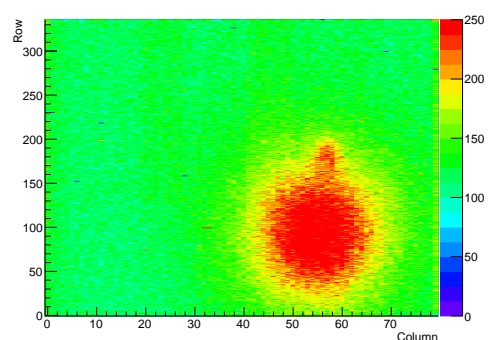

(c)

Figure 1: Noise measurement after to 288 bunches with $2 \mathrm{~mm}$ beam radius for the 3D module in stable-beam (a) and standby (b) configuration as well for the planar module (c).

tored, given that this is highly dependent on the noise. Figures 2 (a) and (b) present the leakage current as a function of the bias voltage of the 3D module in stable-beam and standby configuration, respectively. Different days, corresponding to the different radiation level, are presented. The leakage current distributions have been normalised to $273 \mathrm{~K}$ to take into account the variations in temperature up to $60^{\circ} \mathrm{C}$. In both 3D modules, an increase of the leakage current was observed after each shot. The leakage current as a function of voltage bias for the planar sensor is presented in Figure 3 (a). The level of leakage current after each shot with $2 \mathrm{~mm}$ beam radius is shown. Thanks to the heat dissipator deployed in 2018 , it was possible to keep constant the temperature on the module, $\sim 36{ }^{\circ} \mathrm{C}$. The relation of the leakage current and the proton fluence has been also investigated. Figure 3 (b) show the linear dependence observed for the planar module, as predicted in the literature. The current related damage rate $(\alpha)$ obtained from a linear fit $(\Delta I=\alpha \cdot V \cdot \Phi$, where $\Delta I$ is different in the leakage current measurement pre- and post-irraditation, $V$ is the volume and $\Phi$ the proton fluence) is about $\sim 10^{-10} \mu \mathrm{A} / \mathrm{cm}$.

\footnotetext{
${ }^{3}$ The proton fluence is defined as the sum of the number of bunches times the protons in each bunch divided by one sigma beam cross-section.

${ }^{4}$ The current of a reverse biased diode is called leakage current.
} 


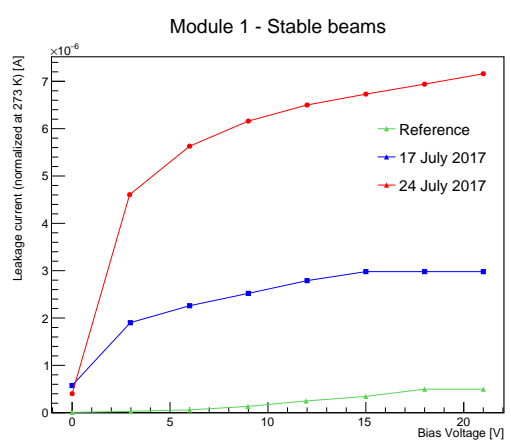

(a)

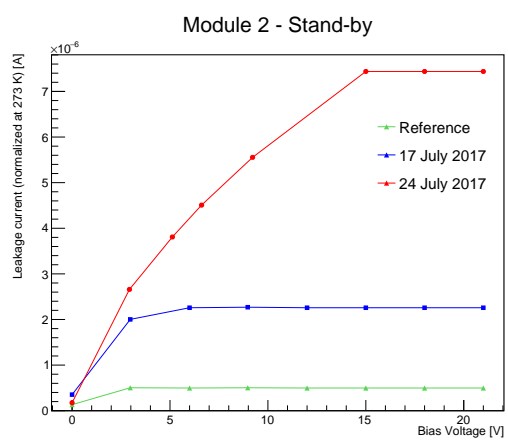

(b)

Figure 2: IV scans performed on 3D modules, (a) module in stable-beam configuraton and (b) module in stand-by configuration, after the beam irradiation: the leakage current increases after irradiation.

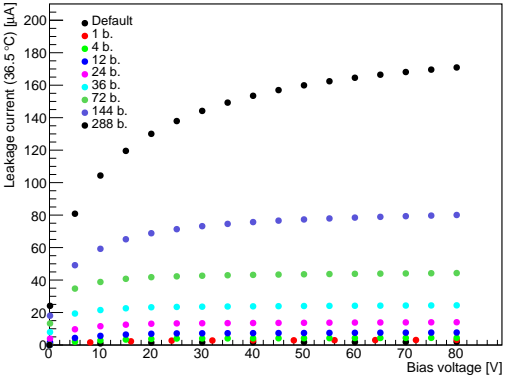

(a)

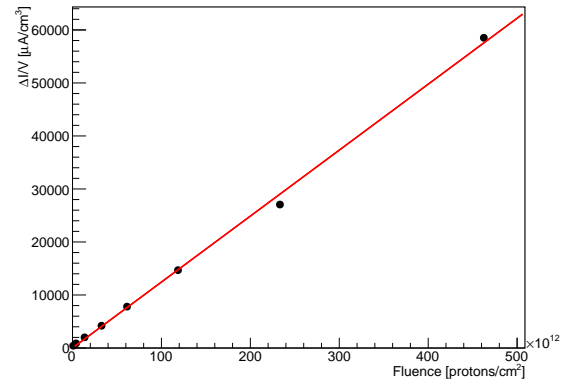

(b)

Figure 3: (a) IV scans performed on planar module after the beam irradiation: the leakage current increases after irradiation, reaching $230 \mu \mathrm{A}$ after 228 bunches. (b) Proton fluence dependence of the leakage current for the pixel module. The red line shows the result of the linear fit of the black points.

\subsection{ITk strip modules}

For the ITk modules, the leakage current was constantly monitored during the beam tests. Due to some technical issue, in 2017 the sensor was not fully depleted. Only a bias voltage of $6 \mathrm{~V}$ was applied to the sensor while in 2018 the sensor was fully depleted (150 V). Figures 4 (a) and (c) show the leakage current values as a function of the time for 2017 and 2018 beam test, respectively. The various vertical lines correspond to the beam shot time. Each color represents a different number of bunches. A clear increase of the leakage current is visible for each shot as well as the relaxation time needed to reach a new stable configuration. The difference between the level of leakage current after each shot and the original value as a function of the proton fluence and Total Ionisating Dose (TID) ${ }^{5}$ is also presented in Figures 4 (b) and (d), for 2017 and 2018 beam

\footnotetext{
${ }^{5}$ Total Ionising Dose is defined as the dose for one proton times the number of protons $\left(T I D=T I D_{1 \mathrm{p}} \times N\right)$. The
} 
test, respectively. A linear fit was performed to obtain a current related damage rate of an order of $10^{-11} \mu \mathrm{A} / \mathrm{cm}$. In 2018 , the read-out system was fully set up and it was possible to perform tests on

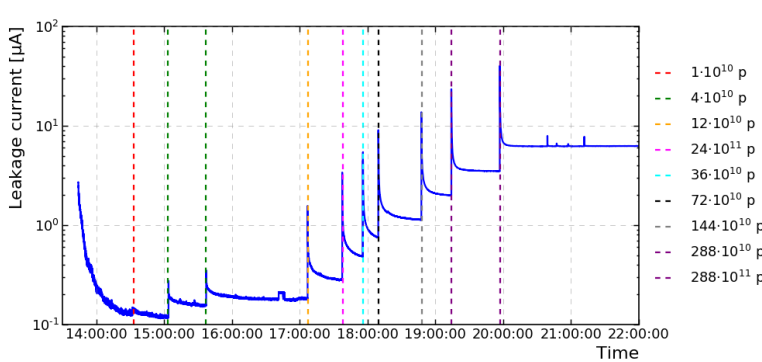

(a)

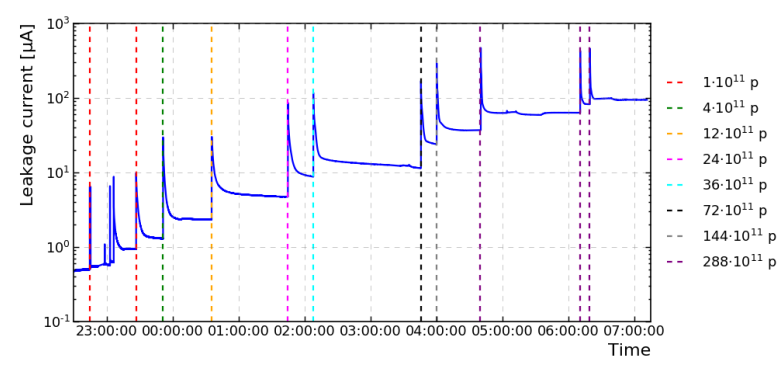

(c)

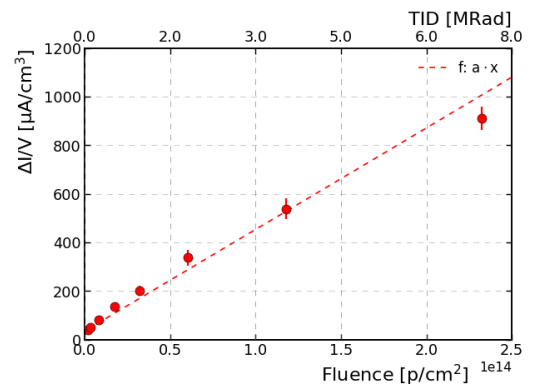

(b)

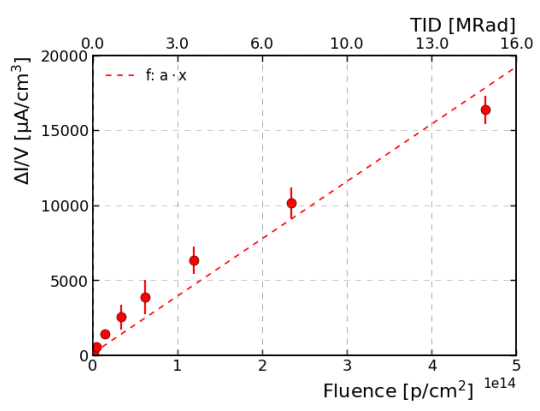

(d)

Figure 4: Leakage current as a function of time for the 2017 (a) and 2018 (c) beam test. Proton fluence and TID dependence of the leakage current for the 2017 (b) and 2018 (d) beam test. The red dashed line shows the result of the linear fit on the red points.

the read-out chip. Through the three points gain test, the noise and gain values per each strip were retrieved after each shot. Figure 5 shows the average noise (a) and average gain (b) over all the strips as a function of the proton fluence and TID. The increase of the noise is concentrated in the first beam shot received by the module. A stable behaviour is reached well before $1 \cdot 10^{13}$ proton $(3$ MRad). With the increase of the proton fluence, a decreasing number of fully operating channels was observed. Using as a role the noise and the gain level, the strips have been classified in the three categories: operational, damaged and noisy (or high-gain). The operational category includes all the strip with noise between 3000 and $40000 \mathrm{ENC}^{6}$ (or gain between 40 and $100 \mathrm{mV} / \mathrm{fC}$ ). The damaged category requires noise lower than $3000 \mathrm{ENC}$ (or gain lower than $40 \mathrm{mV} / \mathrm{fC}$ ). The noisy (or high-gain) category requires noise higher than $40000 \mathrm{ENC}$ (or gain higher than $100 \mathrm{mV} / \mathrm{fC}$ ). The fraction of the channels, belonging in those three categories, has been monitored as a function

number of protons is the sum over the beam shots received by the moduled of the number of bunches times the number of protons in the bunch $\left(N=\sum_{i \in[1,288]} n_{b u n c h}^{i} \cdot 10^{11} \mathrm{p}\right.$. The TID for 1 proton is defined as $T I D_{1 \mathrm{p}}=\frac{d E}{\rho \cdot d x \cdot d S}$, where $\frac{d E}{\rho \cdot d x}$ is assumed to be $2 \mathrm{MeVg}^{-1} \mathrm{~cm}^{2}$ and $d S$ is the beam cross-section.

${ }^{6}$ The high level of noise observed during the operation with respect to nominal noise level reported for similar modules [4] seems to be due electronic circuit or issues encountered after the installation in the facility. A global rescaling needs to be considered for a fair comparison. 


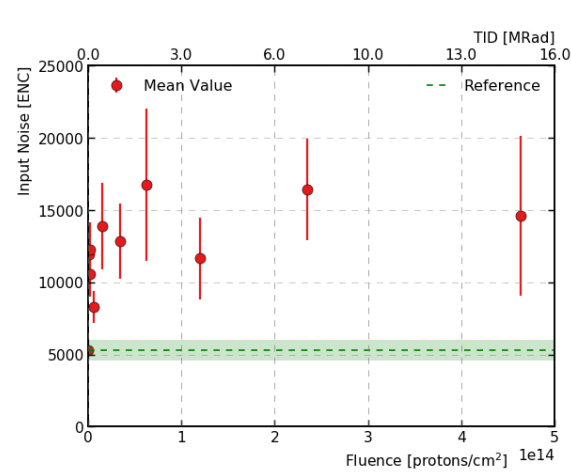

(a)

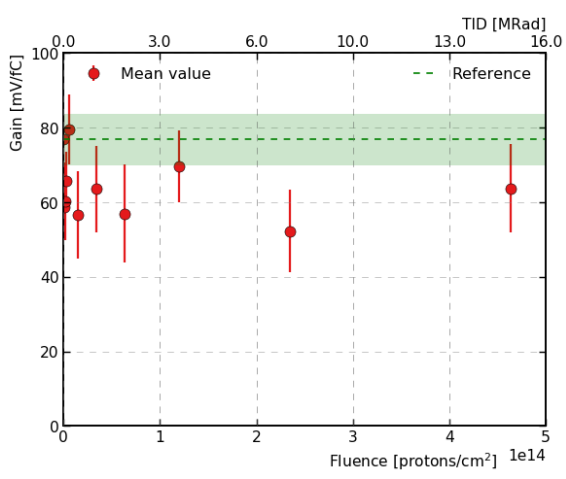

(b)

Figure 5: Proton fluence and TID dependence of the average noise (a) and gain (b). The error bars show the standard deviation of the noise and gain distributions. The green dashed line and the green band represent the mean value and the standard deviation of the noise and gain before irradiation.

of the proton fluence and TID. Figure 6 displays the proton fluence and TID dependence of the fraction of operational, damaged, and noisy (or high-gain) channel using noise (a) and gain (b) level. Similar results are observed with noise or gain classification. After about $6 \cdot 10^{13}$ proton (15 MRad) only $\sim 40 \%$ of the channels are fully working and more than $50 \%$ have been damaged. To

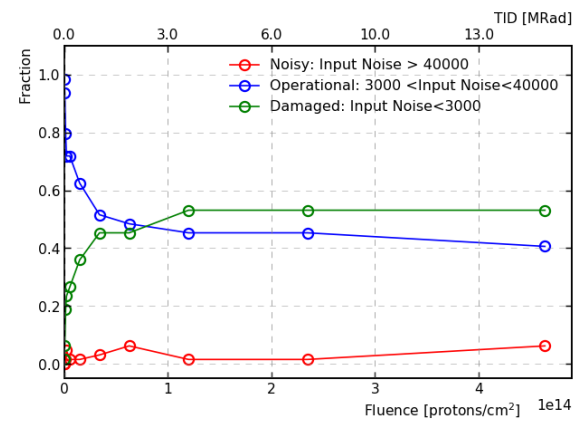

(a)

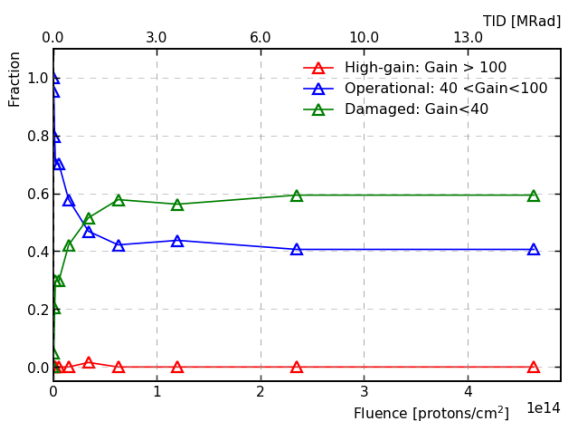

(b)

Figure 6: Proton fluence and TID dependence of the fraction of operational (blue), damaged (green), and noisy (or high-gain) (red) channels. Noise level is used for the classification in Figure (a) while gain in Figure (b).

further investigate the channel behaviour with an increased radiation level, the sensor surface was divided into three slices. The first slice covers the surface closer to the beam centre, around $1 \mathrm{~mm}$ in each side $([0,1] \mathrm{mm}$, where 0 corresponds to the beam centre) which includes 26 strips. The second slice starts at $1 \mathrm{~mm}$ from the beam centre up to $2 \mathrm{~mm}([1,2] \mathrm{mm})$ and includes 24 strips. The third one starts at $2 \mathrm{~mm}$ and includes the remaining sensor surface ([2, rest] $\mathrm{mm}$ ) and strips (14). Figure 7 presents the strips classification as explained before but in each of the three sensor slices. It can be observed that the level of damaged strips is higher in the first slice, $\sim 70 \%$, and decreases in the third part going down to $\sim 20 \%$. From this, we can conclude that there is a higher 
probability to have fully operating channels in the region at least $2 \mathrm{~mm}$ far away from the beam centre with $\sim 80 \%$ of the channels working after about $6 \cdot 10^{13}$ protons (15 MRad).
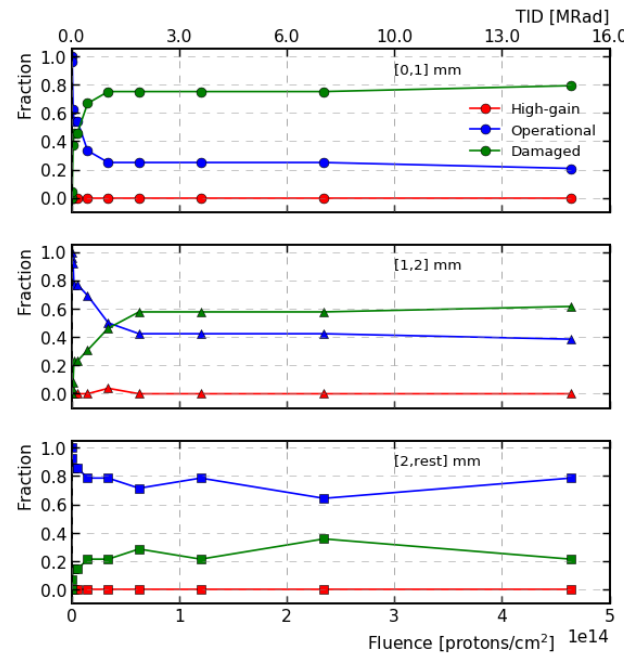

Figure 7: Proton fluence and TID dependence of the fraction of operational (blue), damaged (green), and noisy (red) channels in three slices of the sensor: around the beam centre $([0,1]$ $\mathrm{mm}$, top), after $1 \mathrm{~mm}([1,2] \mathrm{mm}$, middle), and far away ([2, rest $] \mathrm{mm}$, bottom).

\section{Conclusion}

HiRadMat facility was used to irradiate ATLAS silicon detectors with an unprecedented beam intensity. Three pixel IBL modules, with different silicons sensor structures, were operated with stable-beam and/or standby configurations. The noise increase behaves in a similar way for both $3 \mathrm{D}$ and planar sensors. Using the 288 bunches wide ( $2 \mathrm{~mm}$ radius) beam operation with $1 \cdot 10^{11}$ protons/bunch, a conservative estimation of the damage threshold of the pixel modules can be estimated to be $\sim 10^{13}$ protons $/ \mathrm{cm}^{2}$. The value was obtained with beam hitting the modules, sensors and read-out chip, perpendicularly. In 2017, the shot with a 288 bunches narrow $(0.5 \mathrm{~mm}$ radius) beam caused a damaged of the FE-I4 on both operated modules. Detailed inspections indicated the presence of a short circuite between ground and analog voltage in the read-out chip. In 2018, no failures were observed on the sensor and read-out chip after the full irradiation.

For the ITk strip modules, it was observed that the increase in leakage current follows the increase of beam intensity and that the noise level increases as a function of the proton fluence. The fraction of fully operating channels decreases with the increase of the dose received by the sensor. The effect seems to be enhanced around the beam centre and is mitigated with the distance. Since about $10 \%$ of damaged channels was observed after 1 bunch wide beam operation with $1 \cdot 10^{11}$ protons/bunch, a rough estimation of the damage threshold of the strip module was perfomed and found to be $\sim 8 \cdot 10^{11}$ protons $/ \mathrm{cm}^{2}$. The value was calculated using 1 sigma beam spot with the beam hitting the sensor perpendicularly. No macroscopic or physics damages on the sensor and chip were visible after the full irradiation. Further studies are on-going to improve the channel postirradiation characterization. The preliminary outcome indicates that the damages are concentrated on the read-out electronic chip proving the beneficial effect of the PTP mecanism on the strips. 


\section{References}

[1] ATLAS Collaboration, The ATLAS Experiment at the CERN Large Hadron Collider, JINST 3 (2008) S08003.

[2] A. Andreazza, K. Einsweiler, C. Gemme, L. Rossi and P. Sicho, Effect of accidental beam losses on the ATLAS pixel detector, Nucl. Instrum. Meth. A 565 (2006) 50.

[3] G. Apollinari, I. Béjar Alonso, O. Brüning, M. Lamont and L. Rossi, High-Luminosity Large Hadron Collider (HL-LHC): Preliminary Design Report, CERN Yellow Reports: Monographs. CERN, Geneva, 2015.

[4] ATLAS Collaboration, Technical Design Report for the ATLAS Inner Tracker Strip Detector, Tech. Rep. CERN-LHCC-2017-005. ATLAS-TDR-025, CERN, Geneva, Apr, 2017.

[5] ATLAS Collaboration, Technical Design Report for the ATLAS Inner Tracker Pixel Detector, Tech. Rep. CERN-LHCC-2017-021. ATLAS-TDR-030, CERN, Geneva, Sep, 2017.

[6] C. Bertella, C. Escobar, G. Gariano, A. Gaudiello, C. Gemme, S. Katunin et al., Study of damages induced on ATLAS Silicon by fast extracted and intense proton beam irradiation, Nucl. Instrum. Meth. $A$ (2018) .

[7] I. Efthymiopoulos, C. Hessler, H. Gaillard, D. Grenier, M. Meddahi, P. Trilhe et al., HiRadMat: A New Irradiation Facility for Material Testing at CERN, Tech. Rep. CERN-ATS-2011-232, CERN, Geneva, Nov, 2011.

[8] C. D. Via, M. Boscardin, G.-F. D. Betta, G. Darbo, C. Fleta, C. Gemme et al., 3D silicon sensors: Design, large area production and quality assurance for the ATLAS IBL pixel detector upgrade, Nucl. Instrum. Meth. A 694 (2012) 321.

[9] C. Goessling, R. Klingenberg, D. Muenstermann, A. Rummler, G. Troska and T. Wittig, Planar n+-in-n silicon pixel sensors for the ATLAS IBL upgrade, Nucl. Instrum. Meth. A 650 (2011) 198.

[10] ATLAS Collaboration collaboration, ATLAS Insertable B-Layer Technical Design Report, Tech. Rep. CERN-LHCC-2010-013. ATLAS-TDR-19, Sep, 2010.

[11] M. Garcia-Sciveres, D. Arutinov, M. Barbero, R. Beccherle, S. Dube, D. Elledge et al., The FE-I4 pixel readout integrated circuit, Nucl. Instrum. Meth. A 636 (2011) S155.

[12] Y. Unno, S. Edwards, S. Pyatt, J. Thomas, J. Wilson, J. Kierstead et al., Development of n+-in-p large-area silicon microstrip sensors for very high radiation environments - ATLAS12 design and initial results, Nucl. Instrum. Meth. A 765 (2014) 80.

[13] Y. Unno, A. Affolder, P. Allport, R. Bates, C. Betancourt, J. Bohm et al., Development of n-on- $p$ silicon sensors for very high radiation environments, Nucl. Instrum. Meth. A 636 (2011) S24.

[14] N. Lehmann, Tracking with self-seeded Trigger for High Luminosity LHC, Tech. Rep. École Polytechnique Fédérale de Lausanne, Master's thesis, Section of Electrical and Electronical Engineering, 2014. 